ARTICLE

DOI: $10.1038 / s 41467-018-03378-7$

\title{
Current-driven dynamics and inhibition of the skyrmion Hall effect of ferrimagnetic skyrmions in GdFeCo films
}

Seonghoon Woo (1) ${ }^{1}$, Kyung Mee Song ${ }^{1,2}$, Xichao Zhang (1) ${ }^{3}$, Yan Zhou ${ }^{3}$, Motohiko Ezawa (1) ${ }^{4}$, Xiaoxi Liu (10 ${ }^{5}$, S. Finizio ${ }^{6}$, J. Raabe (1) ${ }^{6}$, Nyun Jong Lee ${ }^{7}$, Sang-II Kim7, Seung-Young Park ${ }^{7}$, Younghak Kim ${ }^{8}$, Jae-Young Kim ${ }^{8}$, Dongjoon Lee ${ }^{1,9}$, OukJae Lee ${ }^{1}$, Jun Woo Choi ${ }^{1,10}$, Byoung-Chul Min (1) ${ }^{1,10}$, Hyun Cheol Koo (i) ${ }^{1,9}$ \& Joonyeon Chang ${ }^{1,10}$

Magnetic skyrmions are swirling magnetic textures with novel characteristics suitable for future spintronic and topological applications. Recent studies confirmed the roomtemperature stabilization of skyrmions in ultrathin ferromagnets. However, such ferromagnetic skyrmions show an undesirable topological effect, the skyrmion Hall effect, which leads to their current-driven motion towards device edges, where skyrmions could easily be annihilated by topographic defects. Recent theoretical studies have predicted enhanced current-driven behavior for antiferromagnetically exchange-coupled skyrmions. Here we present the stabilization of these skyrmions and their current-driven dynamics in ferrimagnetic GdFeCo films. By utilizing element-specific X-ray imaging, we find that the skyrmions in the $\mathrm{Gd}$ and FeCo sublayers are antiferromagnetically exchange-coupled. We further confirm that ferrimagnetic skyrmions can move at a velocity of $\sim 50 \mathrm{~m} \mathrm{~s}^{-1}$ with reduced skyrmion Hall angle, $\left|\theta_{\text {SkHE }}\right| \sim 20^{\circ}$. Our findings open the door to ferrimagnetic and antiferromagnetic skyrmionics while providing key experimental evidences of recent theoretical studies.

\footnotetext{
${ }^{1}$ Center for Spintronics, Korea Institute of Science and Technology, Seoul, 02792 Korea. ${ }^{2}$ Department of Physics, Sookmyung Women's University, Seoul, 04130 Korea. ${ }^{3}$ School of Science and Engineering, The Chinese University of Hong Kong, Shenzhen, 518172 China. ${ }^{4}$ Department of Applied Physics, University of Tokyo, Hongo 7-3-1, Tokyo, 113-8656 Japan. ${ }^{5}$ Department of Electrical and Computer Engineering, Shinshu University, Wakasato 4-17-1, Nagano, 380-8553 Japan. ${ }^{6}$ Swiss Light Source, Paul Scherrer Institut, 5232 Villigen, Switzerland. ${ }^{7}$ Spin Engineering Physics Team, Division of Scientific Instrumentation, Korea Basic Science Institute, Daejeon, 305-806 Korea. ${ }^{8}$ Pohang Accelerator Laboratory, Pohang University of Science and Technology, Pohang, 37673 Korea. ${ }^{9}$ KU-KIST Graduate School of Converging Science and Technology, Korea University, Seoul, 02481 Korea. ${ }^{10}$ Department of Nanomaterials Science and Engineering, Korea University of Science and Technology, Daejeon, 34113 Korea. These authors contributed equally: Seonghoon Woo, Kyung Mee Song. Correspondence and requests for materials should be addressed to S.W. (email: shwoo_@kist.re.kr)
} 
M agnetic skyrmions are non-trivial topological objects 1,2 that have been greatly highlighted recently, mainly due to their unique and fascinating topological characteristics suitable for future spintronic applications, such as skyrmion-based racetrack memory ${ }^{3-5}$. Magnetic skyrmions can be stabilized in the presence of a strong Dzyaloshinskii-Moriya interaction $(\mathrm{DMI})^{6,7}$, which prefers non-collinear spin orientation between neighboring magnetic moments. Recent investigations have revealed that in structures where ultrathin ferromagnets are interfaced with large spin-orbit coupling materials, such as $\mathrm{Ta} / \mathrm{CoFeB} / \mathrm{MgO}^{8}, \mathrm{Pt} / \mathrm{Co} / \mathrm{Ir}^{9}, \mathrm{Pt} / \mathrm{Co} / \mathrm{MgO}^{10}, \mathrm{Pt} /$ $\mathrm{Co} / \mathrm{Ta}$, and $\mathrm{Pt} / \mathrm{CoFeB} / \mathrm{MgO}^{11}{ }^{12}$, the interfacial DMI can be strong enough to stabilize the chiral skyrmion structure even at room temperature. It has also been shown that skyrmions can move along magnetic tracks upon the injection of an electrical current $^{8}, 11,13,14$, which indicates that the skyrmions can be adopted in practical devices ${ }^{5}$. However, contrary to theoretical predictions, ferromagnetic skyrmions have shown relatively slow and pinning-dominated current-driven dynamic behaviors at room temperature ${ }^{8,11}, 13-15$. More seriously, ferromagnetic skyrmions exhibit an inevitable topology-dependent effect, namely, the skyrmion Hall effect ${ }^{16-19}$, where the magnetic skyrmions do not move collinear to the current flow direction, but acquire a transverse motion due to the appearance of a topological Magnus force acting upon the non-zero topological charge. The Magnus force is opposite for a ferromagnetic skyrmion whose topological charge $(Q)$ is either $Q=1$ or -1 . To avoid this issue, theoretical studies have suggested that the skyrmion Hall effect can be suppressed by utilizing antiferromagnetically exchange-coupled skyrmions with $Q=1$ and -1 in antiferromagnetic materials ${ }^{20-}$ 23 . Along with the recent interest in antiferromagnets resulting from their intrinsic ultrafast dynamics and insensitivity to disturbing magnetic fields ${ }^{24}$, these advantages in skyrmion dynamics have generated an intense interest in antiferromagnetic skyrmion textures. However, since such an excitation behaves as if it were a simple bubble with $Q=0$, it is quite difficult to experimentally verify if it is really a topological object.

In this work we investigate a similar object in ferrimagnet multilayers. Namely, we consider skyrmion excitations in one magnetic layer and in the magnetic other layer. Since they are antiferromagnetically coupled, their topological charges are opposite. Let us refer to such a pair of skyrmions as a ferrimagnetic skyrmion. The distinctive nature is the topological Magnus forces are not balanced exactly because the magnetization is different between two layers. We predict that ferrimagnetic skyrmions show a small, but non-zero skyrmion Hall effect, which we may use as a verification of the topological excitation. We note a recent study reporting the stabilization of ferrimagnetic skyrmions in $\mathrm{Fe} / \mathrm{Gd}$ multilayers ${ }^{25}$, in which Bloch-type skyrmions are stabilized by long-range dipolar interactions. However,

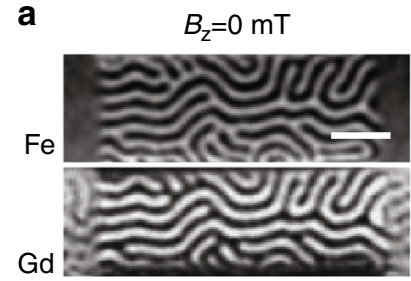

$-100 \mathrm{mT}$

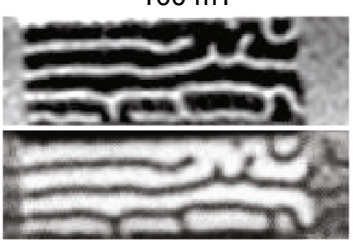

b

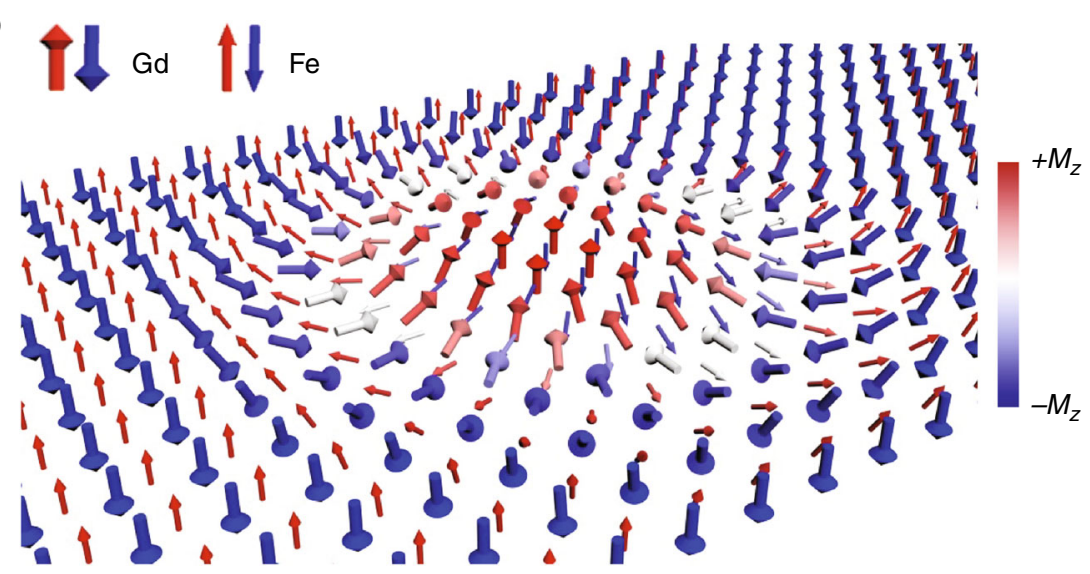

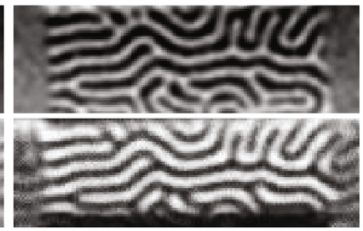

$-120 \mathrm{mT}$

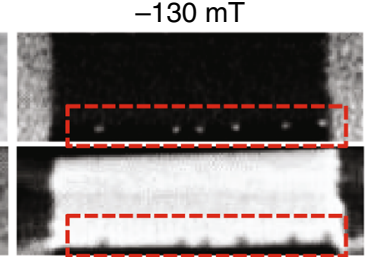

$-70 \mathrm{mT}$

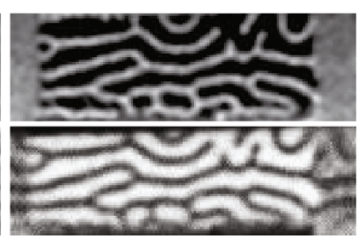

$-130 \mathrm{mT}$
$-50 \mathrm{mT}$

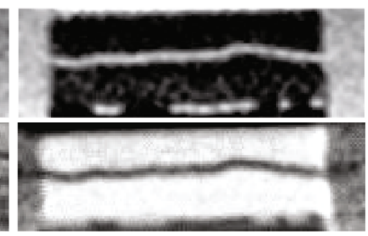

Fig. 1 Scanning transmission X-ray microscopy (STXM) imaging of domain structure upon magnetic field application. a STXM images acquired by sweeping the external perpendicular magnetic field from $B_{z}=0 \mathrm{mT}$ to $B_{z}=-130 \mathrm{mT}$. Dark and bright contrasts correspond to magnetization oriented up (along $+z$ ) and down (along $-z$ ), respectively. Upper panel and lower panel show corresponding images acquired at the $L_{3}$ and $M_{5}$ absorption edges of Fe and $\mathrm{Gd}$, respectively. Note that, due to longer penetration depth associated with the higher energy used for $\mathrm{Gd}$, $\sim 189 \mathrm{eV}$, compared with that of Fe, 709 $\mathrm{eV}$, magnetic contrast under Au electrodes is visible for Gd magnetic moment imaging. b Schematic of antiferromagnetically exchange-coupled ferrimagnetic skyrmion on a magnetic track as observed in our GdFeCo films as indicated in the red dashed-square boxes in the last image of a. Scale bar, 1 $\mu \mathrm{m}$ 
current-driven dynamics of these skyrmions cannot be used for actual applications, mainly due to the random chirality of each individual skyrmion that results in a complex and random current-driven motion ${ }^{26}, 27$. Thus, the actual observation of deterministic and efficient current-driven dynamics of chiral ferrimagnetic skyrmions remains elusive so far and needs to be pursued for the realization of skyrmion-based memory and logic computing devices.

\section{Results}

X-ray microscopy observation of magnetic domains. In our multilayer stack, the Pt heavy-metal underlayer is used to induce a strong DMI that stabilizes chiral magnetic textures. Note that the $\left[\mathrm{Pt}(3 \mathrm{~nm}) / \mathrm{Gd}_{25} \mathrm{Fe}_{65.6} \mathrm{Co}_{9.4}(5 \mathrm{~nm}) / \mathrm{MgO}(1 \mathrm{~nm})\right]_{\mathrm{n}}$ structure with a large repetition number, $n=20$, is used in this study, due to: i) the sizable internal demagnetization field that effectively drives as-grown the magnetization state into the multi-domain state (see Supplementary Fig. 1 and Supplementary Note 1 for its hysteresis behaviors and the top left panel in Fig. 1a for its asgrown multi-domain state), ii) a large amount of magnetic material that resulted in the enhanced magnetic contrast in our $\mathrm{X}$-ray transmission measurement, and iii) promising skyrmion characteristics observed in the structure, which will be presented throughout this Article. Using vibrating sample magnetometry (VSM) measurements, we estimate the magnetization compensation point, $T_{M}$, of our GdFeCo film to be $>450 \mathrm{~K}$ (see Supplementary Fig. 2 and Supplementary Note 2 for details). Hence, our magnetic devices remain at an uncompensated ferrimagnetic state

\section{a}

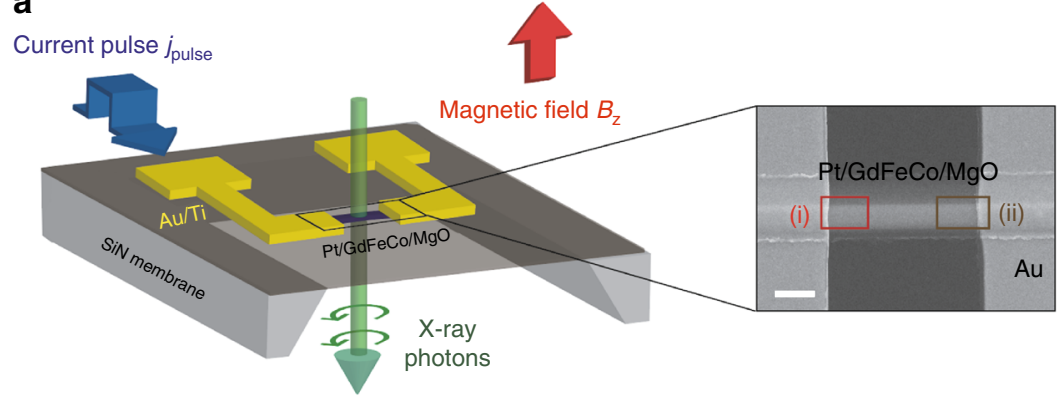

b

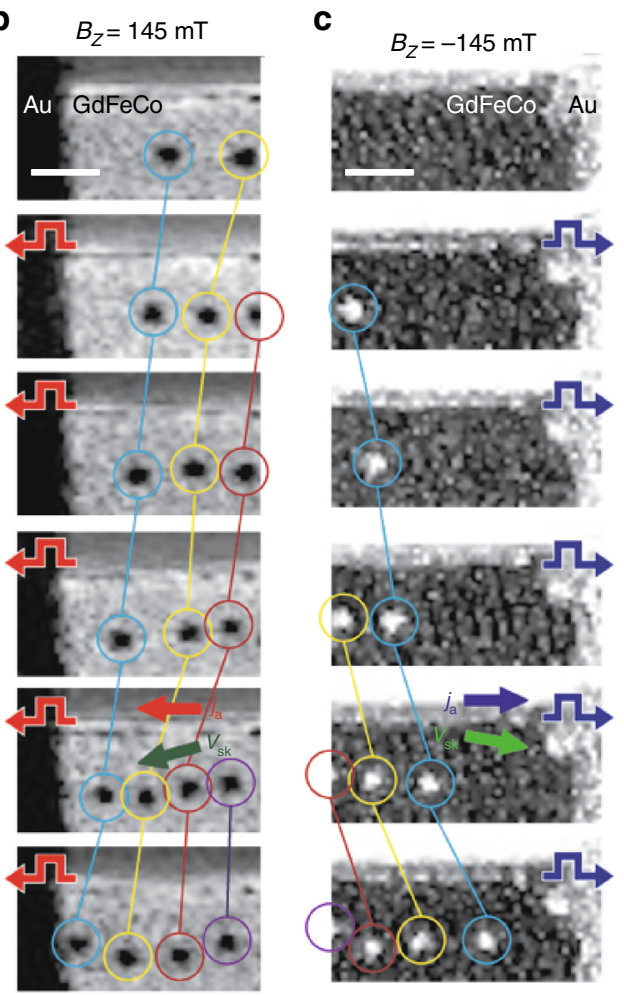

d

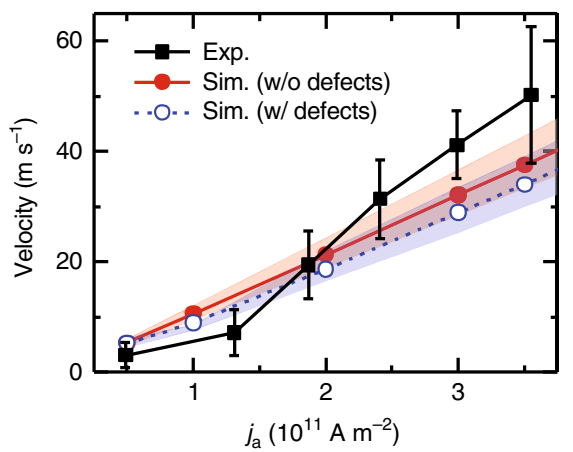

e

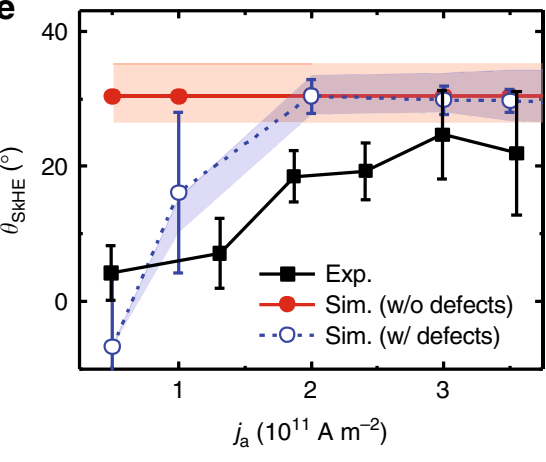

Fig. 2 Current-driven behavior of ferrimagnetic skyrmions and their velocity and skyrmion Hall effect. a Schematic of scanning transmission X-ray microscopy (STXM) geometry, and a scanning electron microscopy (SEM) image of the actual device used for experiments. Scale bar, $2 \mu \mathrm{m}$. Sequential STXM images taken at Fe-edge showing the responses of multiple skyrmions after injecting unipolar current pulses along the track at $\mathbf{b} B_{z}=145 \mathrm{mT}$ and $\mathbf{c} B_{z}=-145 \mathrm{mT}$, respectively. With a fixed pulse-length of single pulse, $5 \mathrm{~ns}$, the pulse amplitude is changed between $4.90 \times 10^{10} \mathrm{~A} \mathrm{~m}^{-2} \leq\left|j_{\mathrm{a}}\right| \leq 3.55 \times 10^{11} \mathrm{~A} \mathrm{~m} \mathrm{~m}^{-2}$. Pulse polarities are indicated as red- and blue-colored arrows inside each image. Within STXM images, the same skyrmion is indicated with the same color. Scale bars, $500 \mathrm{~nm}$. d Experimental and simulated average skyrmion velocity of $\mathrm{Pt} / \mathrm{GdFeCo} / \mathrm{MgO}$ versus current density. e Experimental and simulated average skyrmion $\mathrm{Hall}$ angle of $\mathrm{Pt} / \mathrm{GdFeCo} / \mathrm{MgO}$ versus current density. Experimentally measured skyrmion Hall angles were converted into their absolute value. In $\mathbf{d}$, e The shaded areas in plots represent the simulation results considering the damping coefficient error ranges of $\alpha=0.205 \pm 0.035$, which was measured experimentally as described in Supplementary Fig. 3 and Supplementary Note 3. Note that pulse current densities above $\sim 4 \times 10^{11} \mathrm{~A} \mathrm{~m}^{-2}$ led the damage of the Au contact, which eventually limited the maximum applicable current to our sample. Error bars denote the standard deviation of multiple measurements 
throughout all the room-temperature X-ray measurements. Moreover, to correctly extract the magnetic parameters of our ferrimagnetic films, we have performed several experiments including ferromagnetic resonance (FMR), spin-torque FMR (STFMR), and X-ray magnetic circular dichroism (XMCD) spectroscopy measurements on a companion GdFeCo film grown on $\mathrm{SiO}_{\mathrm{x}} / \mathrm{Si}$ substrate (see Supplementary Figs. 3-6, Supplementary Note 3 and Methods for detailed measurement descriptions and acquired parameter values).

To reveal the nature of ferrimagnetic skyrmions in our system, we first performed element-specific scanning transmission X-ray microscopy (STXM) in the presence of an external perpendicular magnetic field, $B_{z}$. Figure 1a shows the STXM images of the domain structure in a patterned $2.5-\mu \mathrm{m}$-wide and $5-\mu \mathrm{m}$-long $\mathrm{Pt} /$ $\mathrm{GdFeCo} / \mathrm{MgO}$ film with decreasing external magnetic field from $B_{z}=0 \mathrm{mT}$ to $B_{z}=-130 \mathrm{mT}$. The upper and lower panels show corresponding STXM images taken at the absorption edges of Fe ( $\mathrm{L}_{3}$-edge) and $\mathrm{Gd}\left(\mathrm{M}_{5}\right.$-edge), respectively. In these STXM images, dark and bright contrasts correspond to upward $\left(+M_{z}\right)$ and downward $\left(-M_{z}\right)$ magnetization directions, respectively. At $B_{z}=$ $0 \mathrm{mT}$, a labyrinth stripe domain state with the average domain width of $\sim 220 \mathrm{~nm}$ is achieved. Moreover, it is immediately obvious that STXM images at Fe- and Gd-absorption edges show opposite contrast, revealing their expected antiferromagnetic spin ordering within the GdFeCo alloy. Note that, since the measurements are conducted at room temperature, which is lower than the compensation point $T_{M}$, the magnetic moment of Gd aligns parallel with the external magnetic field while the moment of $\mathrm{Fe}$ aligns in an anti-parallel fashion ${ }^{28}$. As the magnetic field increases, fewer domains remain and magnetic configurations become less complex. Eventually, by reaching a magnetic field of $B_{z}=-130 \mathrm{mT}$, we observe multiple isolated skyrmions, and it is evident that the $\mathrm{Gd}$ and Fe magnetic moments are still antiferromagnetically exchange-coupled within these skyrmions, therefore confirming that we observed ferrimagnetic skyrmions. The high spatial resolution $(\sim 25 \mathrm{~nm})$ of STXM allows us to measure the diameter of the observed skyrmions as discussed in Supplementary Figs. 7-8 and Supplementary Note 4 , and we find that the average skyrmion diameter is roughly $\sim 180 \mathrm{~nm}$, which is as small as the skyrmions found in ferromagnetic multilayers with a large DMI value, $1.5 \sim$ $2 \mathrm{~mJ} \mathrm{~m}^{-2}$, studied in refs. ${ }^{9,11,12}$. We later confirmed that our skyrmions exhibited a left-handed Néel-type chirality by observing their current-driven behaviors. Figure $1 \mathrm{~b}$ schematically illustrates the orientation of the antiferromagnetically exchangecoupled internal magnetic moments within the observed ferrimagnetic skyrmion structure.

Current-driven behaviors of ferrimagnetic skyrmions. Having established that ferrimagnetic skyrmions can form at a finite external field in this material, we next studied their currentinduced dynamics in the magnetic track. Figure $2 \mathrm{a}$ shows a schematic drawing of our ferrimagnetic track and electric contacts patterned on a 100 -nm-thick $\mathrm{Si}_{3} \mathrm{~N}_{4}$ membrane for transmission X-ray measurements. The actual scanning electron microscopy (SEM) micrograph of our device is also included, and two indicated areas within the image, (i) and (ii), are used to analyze current-driven skyrmion behaviors shown in Fig. 2b, c, respectively. In Fig. 2b, c, each STXM image, taken at Fe-edge, was acquired after injecting a single current pulse, with the various pulse amplitudes of between $4.90 \times 10^{10} \mathrm{~A} \mathrm{~m}^{-2} \leq\left|j_{\mathrm{a}}\right| \leq$ $3.55 \times 10^{11} \mathrm{~A} \mathrm{~m}^{-2}$ and the pulse duration of $5 \mathrm{~ns}$ (see Supplementary Fig. 9 and Supplementary Note 5 for details on the electronic connections and the actual pulse shape). Note that each skyrmion is color-circled, and a single color is used for the same skyrmion throughout each sequence. The pulse polarity, defined by the electric current flow direction, is indicated in the figure as red and blue pulse-shaped arrows. It is also noteworthy that while skyrmion core points $+z$ and $-z$ directions in STXM images in Fig. $2 b, c$, respectively, the effective core magnetization points $-z$ and $+z$ directions in Fig. 2b, c, respectively, because Gd moments are dominant in our material at room temperature as discussed above.

Figure $2 b$ first shows a sequence of STXM images of skyrmions stabilized by a magnetic field of $B_{z}=145 \mathrm{mT}$ near the left $\mathrm{Au}$ electrode on a magnetic track. In our field of view, there are initially two skyrmions, and as we inject leftward current pulses, additional two skyrmions appear while all of the skyrmions show homogeneous propagations. Moreover, it is noticeable that, when a train of the skyrmions propagates along the track, their alignment/trajectory shows a finite angle with respect to the current flow direction, which is the hallmark of the skyrmion Hall effect. We then reversed the magnetic field to $B_{z}=-145 \mathrm{mT}$ and investigated another region near the right $\mathrm{Au}$ electrode on the same track, as shown in Fig. 2c. While no skyrmion is observed in the field of view of the first image, up to four skyrmions appear with pulse injections, and all of them show pinning-free homogeneous displacements, as was observed for the other polarity of skyrmions in Fig. $2 \mathrm{~b}$. Note that a train of these bright skyrmions also shows transverse velocity component, and surprisingly, the sign of slope is opposite to the case of dark skyrmions. This is due to the opposite topological polarity of skyrmions for two cases, which experience the opposite sign of the topological Magnus force that consequently provides opposite transverse propagation directions. This observation of finite skyrmion Hall effect and the symmetry of skyrmion Hall angle in our ferrimagnetic material agrees well with the cases of ferromagnetic skyrmions in $\mathrm{Ta} / \mathrm{CoFeB} / \mathrm{TaO}_{\mathrm{x}}{ }^{17}$ and $\mathrm{Pt} / \mathrm{CoFeB} /$ $\mathrm{MgO}^{18}$. Moreover, in the last image of each sequence, we observe the repulsion between skyrmions and the edges of the sample, which locates right underneath the field of views as shown in Fig. 2a. The repulsion occurs due to the DMI boundary condition $^{29}$, which results in the skyrmion motion (i) back toward the sample center (blue-circled skyrmion in Fig. 2b) or (ii) straight along the sample edge (yellow-circled skyrmion in Fig. 2b and blue-circled skyrmion in Fig. 2c), which also agrees with previously observed ferromagnetic skyrmion motion along the edge in Ref. ${ }^{17}$.

With these observations shown in Fig. 2b, c, three important qualitative conclusions on the skyrmion physics within ferrimagnetic material can be drawn. First and most importantly, our investigation reveals that ferrimagnetic skyrmions can also be displaced by electric currents at room temperature just as the ferromagnetic skyrmions $8,11-14,17,18$. Moreover, we show that the skyrmion propagation direction is along the current flow direction (against the electron flow) for both $+M_{z}$-core and $-M_{z}$-core skyrmions, and this same directionality agrees well with the spin Hall current-driven motion of homochiral left-handed Néel-type hedgehog skyrmions stabilized by interfacial DMI in $\mathrm{Pt} /$ ferromagnet thin films ${ }^{11-14,18}$. This implies that the interfacial DMI at the Pt/ GdFeCo interface plays a crucial role in stabilizing skyrmions and also driving them on the track in our ferrimagnetic structure. Furthermore, the skyrmion pinning, which was often observed in many of ferromagnetic systems ${ }^{11,13,14,17}$, is significantly reduced in our ferrimagnetic material. This low pinning may originate from the amorphous nature of $\mathrm{GdFeCo}$ because the absence of grain boundaries leads to lower skyrmion pinning, as was observed in amorphous $\mathrm{CoFeB}$ ferromagnetic films ${ }^{11}$. Overall, it is noteworthy that our observation serves as the first experimental observation of 
current-driven excitation of nanoscale magnetic skyrmions in ferrimagnets.

The pulse amplitude-dependent skyrmion velocity and its skyrmion Hall angle are plotted in Fig. 2d, e, respectively. To correctly calculate the distance and angle between two images, we have performed image-displacement correction using the edge between our magnetic track and Au electrode. It is first noticeable that skyrmion velocity increases linearly with pulse amplitudes, and the maximum velocity approaches $\sim 50 \mathrm{~m} \mathrm{~s}^{-1}$ at $\left|j_{\mathrm{a}}\right|=3.55 \times$ $10^{11} \mathrm{~A} \mathrm{~m}^{-2}$, which is comparable to the current state-of-the-art skyrmions observed in a few ferromagnetic heterostructures $^{11}$ 14, 18. Moreover, as shown in Fig. 2e, we observe a very small skyrmion Hall angle, $\left|\theta_{\mathrm{SkHE}}\right|$ up to $\sim 20^{\circ}$, which is far lower than the skyrmion Hall angles, $\left|\theta_{\mathrm{SkHE}}\right|>30^{\circ}$, observed for ferromagnetic skyrmions in $\mathrm{Ta} / \mathrm{CoFeB} / \mathrm{MgO}$ and $\mathrm{Pt} /$ $\mathrm{CoFeB} / \mathrm{MgO}$ structures ${ }^{17,18}$. Antiferromagnetic coupling between two sublayers and the corresponding largely reduced net magnetization within GdFeCo films have led to the effective inhibition of the skyrmion Hall effect. It is noteworthy that relatively large skyrmion Hall effect in conventional ferromagnets may lead skyrmions toward device edges, where they could easily be annihilated by topographic defects ${ }^{20}, 30$. Moreover, the skyrmion Hall effect-driven strong transverse motion may pose a limitation for the maximum skyrmion speed due to the finite edge repulse. Therefore, we believe that our ferrimagnetic multilayers can serve as an important magnetic material for such future skyrmionic devices that could replace conventional ferromagnets with enhanced reliability and mobility. Note that skyrmion Hall angle increases monotonically at low-current densities, $\left|j_{\mathrm{a}}\right|<\sim 2 \times 10^{11} \mathrm{~A} \mathrm{~m}^{-2}$, and saturates at high current densities, because the skyrmion dynamics is dominated by pinning sites at low driving forces, which is similar to the creep motion of ferromagnetic skyrmions in low-current-density regime caused by the pinning potential ${ }^{15,17}$. The remnant finite skyrmion Hall angle, $\left|\theta_{\mathrm{SkHE}}\right| \sim 20^{\circ}$, results from the uncompensated magnetic moments between $\mathrm{Gd}$ and FeCo sublayers at 300 $\mathrm{K}<T_{M}$, where $M_{\mathrm{S}_{-} \mathrm{Gd}} \neq M_{\mathrm{S}_{-} \mathrm{FeCO}}$. (see Methods). Therefore, by adjusting material compositions, it will be possible to further reduce the effective skyrmion Hall angle in ferrimagnetic GdFeCo films.

Micromagnetic simulation on ferrimagnetic skyrmion dynamics. For more comparison, we simulated the currentdriven dynamics of a ferrimagnetic skyrmion (see Methods for more modeling details) in a checkerboard-like two-sublayer spin system based on the G-type antiferromagnetic structure with simple square lattices ${ }^{20}, 30,31$, where the two sublayers, corresponding to $\mathrm{Gd}$ and $\mathrm{FeCo}$, are coupled in a ferrimagnetic manner with a net saturation magnetization, while each sublayer is ferromagnetically ordered. We have also examined simulations using the two-sublattice model with classic $J_{1}-J_{2}-J_{2}{ }^{\prime}$ Heisenberg exchange interactions as shown in Supplementary Figs. 10-12 and Supplementary Note 6. While we find that the intrasublattice exchange interactions indeed affect on the ferrimagnetic skyrmion size and dynamics, however, the influence of these effects on the overall dynamics, especially on the skyrmion Hall effect, turns out not to be significant in both qualitative and quantitative results.

Simulations were performed with both models: with and without pinning defects, using experimentally measured materials parameters given in Methods. Moreover, in simulations, we considered the error range of damping coefficient measurement shown in Supplementary Fig. 3 and Supplementary Note 3, because the ferrimagnetic skyrmion dynamics can be strongly influenced by small changes in damping coefficient (see
Supplementary Figs. 13-14 and Supplementary Note 7 for details). Note that the error ranges of simulations are shown as shaded areas in Figs. 2d, e. The simulated skyrmion velocity as a function of the current density is first shown in Fig. $2 \mathrm{~d}$. Simulation results show qualitative and quantitative agreement with experimental observations, revealing that the ferrimagnetic skyrmion velocity is linearly proportional to the driving current density. We also calculated the skyrmion Hall angle as a function of the current density as shown in Fig. 2e. It is first noticeable that the skyrmion Hall angle in the model without pinning defects is independent of the current density, while the skyrmion Hall angle in the model with certain pinning defects increases with increasing current density and approaches a constant value calculated with the pinning-absent model. This linear increase is qualitatively consistent with our experimental observations and also with previous report ${ }^{17}$, indicating the existence of certain pinning effects due to impurities or defects in the real material. Moreover, the calculated skyrmion Hall angle considering the damping errors shows a fair quantitative agreement with experimental observation.

Although the averaged skyrmion Hall angle for simulation is still slightly larger than experiments, we speculate that the small difference may originate from the larger effective damping associated with skyrmion dynamics. Weindler et al. recently reported that local FMR $(\alpha=0.0072)$ and domain wall dynamics measurements $(\alpha=0.023)$ yield very different damping parameters for the same material, Permalloy, and magnetic textureinduced nonlocal damping may be responsible for the increase in effective damping ${ }^{32}$. Gerrits et al. also reported that, unlike smallangle magnetization dynamics, such as conventional FMR, largeangle magnetization dynamics could induce an increase in the apparent damping ${ }^{33}$, and we believe this scenario could also be used to explain our case, where skyrmion motion involves the large-angle magnetization dynamics. Moreover, because a patterned $2.5-\mu \mathrm{m}$-wide and $5-\mu \mathrm{m}$-long nanowire structure was used for skyrmion study while continuous films were employed in material parameter analysis, roughness-induced extrinsic damping enhancement could be another source of damping increase ${ }^{34}$. Nevertheless, by considering above possible scenarios, our quantitative results on the suppression of the skyrmion Hall effect can be reasonably understood.

\section{Discussion}

We have so far observed that the current-driven behaviors of ferrimagnetic skyrmions are indeed attractive compared with their ferromagnetic counterparts. However, it may also be possible that the small value of skyrmion Hall angle in a ferrimagnetic material is only from the small saturation magnetization in a ferrimagnet and not from its antiferromagnetic characteristic. Thus, for a fair comparison between ferrimagnetic and ferromagnetic skyrmions, we have performed simulations on the current-driven dynamics of skyrmions with the same low (net) saturation magnetization, as shown in Fig. 3. It should first be pointed out that, by using the same material parameters, the sizes of the ferrimagnetic and ferromagnetic skyrmions are measured to be identical at certain out-of-plane magnetic fields (Fig. 3a, inset). Figure 3a shows the velocities of the current-driven ferrimagnetic and ferromagnetic skyrmions as a function of the driving current density. It can be seen that both the ferrimagnetic and ferromagnetic skyrmion velocities increase with increasing driving current density, where the mobility of ferrimagnetic skyrmion is slightly larger than that of ferromagnetic skyrmion. More significantly, as shown in Fig. 3b, the skyrmion Hall angle of ferrimagnetic skyrmion is significantly smaller than that of the ferromagnetic skyrmion by a factor of 2 , even the net saturation 

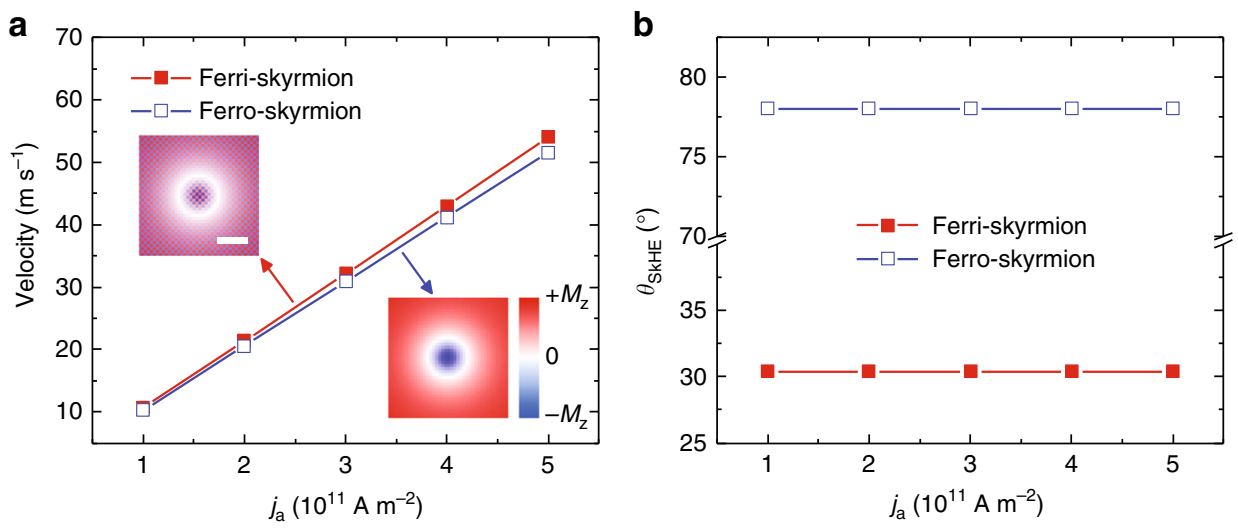

Fig. 3 Comparison between the current-driven dynamics of ferrimagnetic and ferromagnetic skyrmions of the same net saturation magnetization. a The ferrimagnetic and ferromagnetic skyrmion velocities as a function of the driving current density. Insets show the close-up top views of the ferrimagnetic skyrmions in a square film with periodic boundary conditions in both $x$ and $y$ directions. The lattice constant is set as $5 \AA$. $\mathbf{b}$ The ferrimagnetic and ferromagnetic skyrmion Hall angle as a function of the driving current density. The net saturation magnetization was set to be $M_{\mathrm{S}}=2 \times 10^{5} \mathrm{~A} \mathrm{~m}^{-1}$ for both cases. Scale bar, $5 \mathrm{~nm}$

magnetizations are the same for both skyrmions. Note that skyrmion Hall angles are independent of the current densities for both cases, as the pinning effect is not considered in this comparison. To compare the effect of pinning on ferrimagnetic and ferromagnetic skyrmions more carefully, we have simulated the current-driven dynamics of both ferrimagnetic and ferromagnetic skyrmions in the same disorder model, which is shown in Supplementary Fig. 15 and Supplementary Note 8. It is found that the given pinning effect on the current-driven skyrmion dynamics is not significant for both ferrimagnetic and ferromagnetic cases, especially at a large driving current density (e.g., $5 \times 10^{11} \mathrm{~A} \mathrm{~m}^{-2}$ ), however, at a small driving current density (e.g., $1 \times 10^{11} \mathrm{~A} \mathrm{~m}^{-2}$ ), the ferrimagnetic skyrmion motion is more influenced by the pinning effect. The reason could be that the ferromagnetic skyrmion experiences a stronger Magnus force, which helps it in overcoming obstacles ${ }^{35,36}$. Nevertheless, larger skyrmion velocity and smaller skyrmion Hall angle for ferrimagnetic case were maintained over the whole range of examined current densities. Overall, the simulation results indicate that even when the ferrimagnetic and ferromagnetic skyrmions have the same low (net) saturation magnetization, the current-driven dynamics of the ferrimagnetic skyrmion is much more reliable for the transport in narrow channels as information carriers.

In conclusion, we have observed and studied the stabilization and current-driven dynamics of antiferromagnetically exchangecoupled skyrmions in ferrimagnetic GdFeCo films. By utilizing the element-specific X-ray imaging, we have identified that the ferrimagnetic skyrmion in the GdFeCo films consists of two antiferromagnetically exchange-coupled skyrmions in the Gd and FeCo sublayers. We further confirm that current-driven ferrimagnetic skyrmions can move at a velocity of $\sim 50 \mathrm{~m} \mathrm{~s}^{-1}$ with reduced skyrmion Hall angle, $\left|\theta_{\mathrm{SkHE}}\right| \sim 20^{\circ}$. With micromagnetic simulations, we reveal that ferrimagnetic skyrmions are much more attractive than their ferromagnetic counterparts in many technological-relevant aspects, such as larger skyrmion mobility and strongly suppressed skyrmion Hall effect, mainly due to their antiferromagnetic nature. Our findings reveal the promising dynamic properties of ferrimagnetic skyrmions, and highlight the possibility to build more reliable skyrmionic devices using ferrimagnetic and antiferromagnetic materials.

\section{Methods}

Sample preparation and experimental method. The $[\mathrm{Pt}(3 \mathrm{~nm}) /$

$\left.\mathrm{Gd}_{25} \mathrm{Fe}_{65.6} \mathrm{Co}_{9.4}(5 \mathrm{~nm}) / \mathrm{MgO}(1 \mathrm{~nm})\right]_{20}$ films were grown by $\mathrm{DC}$ magnetron sputtering at room temperature under $1 \mathrm{mTorr}$ Ar for Pt and GdFeCo and $4 \mathrm{mTorr} \mathrm{Ar}$ for $\mathrm{MgO}$ at a base pressure of roughly $\sim 2 \times 10^{-8}$ Torr. Samples were grown on a 100 -nm-thick SiN substrate and then patterned using electron beam lithography and lift-off technique. Nominally same films were grown on $\mathrm{SiOx} / \mathrm{Si}$ substrate for VSM, FMR, ST-FMR, asymmetric bubble expansion measurements. The series of measurements yielded material constants: anisotropic field $\mu_{0} H_{\mathrm{k}}=0.15 \mathrm{~T}$, net saturation magnetization $M_{\mathrm{S}}=2 \times 10^{5} \mathrm{~A} \mathrm{~m}^{-1}$, uniaxial anisotropy, $K_{\mathrm{u}}=4.01 \times 10^{4}$ $\mathrm{J} \mathrm{m}^{-3}$, damping coefficient, $\alpha=0.205 \pm 0.035$, spin Hall angle, $\theta_{\mathrm{SH}}=0.055$, magnetization ccompensation ratio, $n=M_{\mathrm{S}}^{\mathrm{FeCo}} / M_{\mathrm{S}}^{\mathrm{Gd}}=0.78$ and DMI magnitude, $D$ $=-0.98 \mathrm{~mJ} \mathrm{~m}^{-2}$ (see Supplementary Note 3 for details).

All microscopy images were acquired using the STXM installed at the PolLux (X07DA) beamline of the Swiss Light Source (SLS) at the Paul Scherrer Institute in Villigen, Switzerland. The device used for the experiments was 2.5 - $\mu$ m-wide and 5- $\mu \mathrm{m}$-long, which yielded an electrical resistance of $\sim 57 \mathrm{Ohms}$ measured in 2 -point. This resistance reduced the impedance mismatch of the device, and allowed an almost complete transmission of 5-ns-long short electrical pulses across the device. This was verified by simultaneously measuring the injected (through a $-20 \mathrm{~dB}$ pickoff $\mathrm{T}$ ) and transmitted samples with a real-time oscilloscope. Pulse current densities above $\sim 4 \times 10^{11} \mathrm{~A} \mathrm{~m}^{-2}$ led to a damage of the Au contact, which eventually limited the maximum current applied in Fig. 2. Skyrmion velocities were determined using the total displacements, measured by acquiring STXM images before and after the injection of the pulses, and the integrated pulse time. Three to ten displacements were recorded for each pulse amplitude, and the average value and standard deviations of the individual velocity measurements are plotted in Fig. $2 \mathrm{~d}$. Current densities were calculated by dividing the injected current with stripe width and effective total thickness of Pt and GdFeCo.

Simulation method. The spin dynamics simulation is carried out by using the Object Oriented MicroMagnetic Framework (OOMMF) with the home-made extension modules for the periodic boundary condition ${ }^{37}$. The model is treated as a checkerboard-like two-sublattice spin system based on the G-type anti-

ferromagnetic structure with simple square lattices, where the two sublattices are coupled in a ferrimagnetic manner with a net spontaneous magnetization, while each sublattice is ferromagnetically ordered. The Hamiltonian is based on the classical Heisenberg model, given as

$$
\begin{aligned}
\mathcal{H}=-J_{i j} \sum_{<i, j>} \mathbf{S}_{i} \cdot \mathbf{S}_{j}-J_{i j}^{\mathrm{A}} & \sum_{\ll i, j\rangle_{\mathrm{A}}} \mathbf{S}_{i} \cdot \mathbf{S}_{j}-J_{i j}^{\mathrm{B}} \sum_{\ll i, j \gg_{\mathrm{B}}} \mathbf{S}_{i} \cdot \mathbf{S}_{j}+D \sum_{<i, j>}\left(\mathbf{u}_{i j} \times \hat{z}\right) \cdot\left(\mathbf{s}_{i} \times \mathbf{S}_{j}\right) \\
& -K \sum_{i}\left(\mathbf{S}_{i}^{z}\right)^{2}-\sum_{i} \mu_{i} \mathbf{S}_{i} \cdot \boldsymbol{H}+H_{\text {DDI }}
\end{aligned}
$$

where $\mathbf{S}_{i}$ represents the local spin vector reduced as $\mathbf{S}_{i}=\boldsymbol{M}_{i} / M_{\mathrm{S}}^{i}$ at the site $i$, and $\mathbf{S}_{j}$ represents the local spin vector reduced as $\boldsymbol{S}_{j}=\boldsymbol{M}_{j} / \boldsymbol{M}_{\mathrm{S}}^{j}$ at the site $j . \boldsymbol{M}_{i}$ and $\boldsymbol{M}_{j}$ are the magnetization at the site $i$ and $j$, respectively. $M_{\mathrm{S}}^{i}$ denotes the saturation magnetization of the sublattice $i$, while the saturation magnetization of sublattice $j$ is defined as $M_{\mathrm{S}}^{j}=n M_{\mathrm{S}}^{i}$ with the compensation ratio $n .<i, j>$ runs over all the nearest-neighbor sites in the two-sublattice spin system. $\ll i, j \gg_{\mathrm{A}}$ and $\ll i, j \gg_{\mathrm{B}}$ run over all the nearest-neighbor sites in the sublattice A and sublattice B, respectively (see Supplementary Fig. 10). $J_{i j}$ is the exchange coupling energy constant between the two adjacent spin vector $S_{i}$ and $S_{j}$, which has a negative value $\left(J_{i j}<0\right)$ representing the antiferromagnetic spin ordering of the two sublattices. $J_{i j}^{\mathrm{A}}$ and $J_{i j}^{\mathrm{B}}$ are the exchange coupling energy constants for the sublattice A and sublattice B, 
respectively, which are positive numbers $\left(J_{i j}^{\mathrm{A}}>0, J_{i j}^{\mathrm{B}}>0\right)$ representing the ferromagnetic intra-sublattice coupling. $D$ is the interface-induced DMI constant, $\mathbf{u}_{i j}$ is the unit vector between spins $\mathbf{S}_{i}$ and $\mathbf{S}_{j}$, and $\hat{z}$ is the interface normal, oriented from the heavy-metal layer to the ferrimagnetic layer. $K$ is the perpendicular magnetic anisotropy (PMA) constant, $\boldsymbol{H}$ is the applied magnetic field, and $H_{\mathrm{DDI}}$ stands for the dipole-dipole interaction, i.e., the demagnetization effect.

The time-dependent dynamics of the spin system is controlled by the Landau-Lifshitz-Gilbert (LLG) equation augmented with the damping-like spin Hall torque, which is expressed as

$$
\frac{\mathrm{d} \mathbf{S}_{i}}{\mathrm{~d} t}=-\gamma_{0} \mathbf{S}_{i} \times \mathbf{H}_{\text {eff }}+\alpha\left(\mathbf{S}_{i} \times \frac{\mathrm{d} \mathbf{S}_{i}}{\mathrm{~d} t}\right)+\tau\left[\mathbf{S}_{i} \times\left(\hat{p} \times \mathbf{S}_{i}\right)\right]
$$

where $\mathbf{H}_{\text {eff }}=-\left(1 / \mu_{0} M_{\mathrm{S}}^{i}\right) \cdot\left(\delta \mathcal{H} / \delta \mathbf{S}_{i}\right)$ is the effective field on a lattice site, $\gamma_{0}$ is the Gilbert gyromagnetic ratio, and $\alpha$ is the phenomenological damping coefficient. The coefficient for the spin Hall torque is given as $\tau=\left(\gamma_{0} \hbar j \theta_{\mathrm{SH}}\right) /\left(2 \mu_{0} e M_{\mathrm{S}}^{i} b\right)$, where $j$ is the applied charge current density, $\theta_{\mathrm{SH}}$ is the spin Hall angle, and $b$ is the thickness of the ferrimagnetic layer. $\hat{p}=\boldsymbol{j} \times \hat{\boldsymbol{z}}$ denotes the spin polarization direction.

For the simulation on the multilayer structure, we employed an effective medium approach ${ }^{11}$ with the lattice constant of $5 \AA$, which improves the computational speed by converting the multilayer into a two-dimensional effective model with reduced parameters. The intrinsic magnetic parameters used in the simulation are measured from our experimental samples as well as adopted from Refs. ${ }^{11,18,38}$ : the damping coefficient $\alpha=0.205 \pm 0.035$, the inter-sublattice exchange stiffness $A_{\mathrm{Gd}-\mathrm{Fe}}=-10 \mathrm{pJ} \mathrm{m}^{-1}, A_{\mathrm{Gd}-\mathrm{Gd}}=5 \mathrm{pJ} \mathrm{m}^{-1}, A_{\mathrm{Fe}-\mathrm{Fe}}=5 \mathrm{pJ} \mathrm{m}^{-1}$, the spin Hall angle $\theta_{\mathrm{SH}}=0.055$, the DMI constant $D=-0.96 \mathrm{~mJ} \mathrm{~m}^{-2}$, the PMA constant $K_{u}=4.01 \times 10^{4} \mathrm{~J} \mathrm{~m}^{-3}$, and the net saturation magnetization $M_{\mathrm{S}}=M_{\mathrm{S}}^{\mathrm{Gd}}-M_{\mathrm{S}}^{\mathrm{Fe}}=200 \mathrm{kAm}^{-1}$. The compensation ratio is measured as $n=M_{\mathrm{S}}^{\mathrm{Fe}} / M_{\mathrm{S}}^{\mathrm{Gd}}=0.78$. In the experimental multilayer system, the thickness of one ferrimagnetic layer is $t_{\mathrm{m}}=5 \mathrm{~nm}$, the thickness of one repetition is $t_{\mathrm{r}}=9 \mathrm{~nm}$, and the number of repetitions is $n_{\text {rep }}=20$. For the simulation on the model with pinning defects, the defects with the size of $10 \AA \times 10 \AA$ and a higher PMA $\left(K_{\mathrm{p}}=\right.$ $5 K_{\mathrm{u}}$ ) are randomly distributed in the whole ferrimagnetic layer. The density of the defects in the whole model equals $5 \%$.

Data availability. Data supporting the findings of this study are available within the article and its Supplementary Information files and from the corresponding author upon request. The micromagnetic simulator OOMMF used in this work is publicly accessible at http://math.nist.gov/oommf.

Received: 13 June 2017 Accepted: 5 February 2018

Published online: 06 March 2018

\section{References}

1. Rößler, U. K., Bogdanov, A. N. \& Pfleiderer, C. Spontaneous skyrmion ground states in magnetic metals. Nature 442, 797-801 (2006).

2. Mühlbauer, S. et al. Skyrmion lattice in a chiral magnet. Science 323, 915-919 (2009).

3. Jonietz, F. et al. Spin transfer torques in $\mathrm{MnSi}$ at ultralow current densities. Science 330, 1648-1651 (2010).

4. Yu, X. Z. et al. Skyrmion flow near room temperature in an ultralow current density. Nat. Commun. 3, 988 (2012).

5. Fert, A., Cros, V. \& Sampaio, J. Skyrmions on the track. Nat. Nanotechnol. 8, 152-156 (2013).

6. Dzyaloshinsky, I. A thermodynamic theory of 'weak' ferromagnetism of antiferromagnetics. J. Phys. Chem. Solids 4, 241-255 (1958).

7. Moriya, T. Anisotropic superexchange interaction and weak ferromagnetism. Phys. Rev. 120, 91-98 (1960).

8. Jiang, W. et al. Blowing magnetic skyrmion bubbles. Science 349, 283-286 (2015).

9. Moreau-Luchaire, C. et al. Additive interfacial chiral interaction in multilayers for stabilization of small individual skyrmions at room temperature. Nat. Nanotechnol. 11, 444-448 (2016)

10. Boulle, O. et al. Room-temperature chiral magnetic skyrmions in ultrathin magnetic nanostructures. Nat. Nanotechnol. 11, 449-454 (2016).

11. Woo, S. et al. Observation of room-temperature magnetic skyrmions and their current-driven dynamics in ultrathin metallic ferromagnets. Nat. Mater. 15, 501-506 (2016).

12. Woo, S. et al. Spin-orbit torque-driven skyrmion dynamics revealed by timeresolved X-ray microscopy. Nat. Commun. 8, 15573 (2017).

13. Legrand, W. et al. Room-temperature current-induced generation and motion of sub-100 nm skyrmions. Nano. Lett. 17, 2703-2712 (2017).

14. Hrabec, A. et al. Current-induced skyrmion generation and dynamics in symmetric bilayers. Nat. Commun. 8, ncomms15765 (2017).
15. Hanneken, C., Kubetzka, A., Bergmann, Kvon \& Wiesendanger, R. Pinning and movement of individual nanoscale magnetic skyrmions via defects. New. J. Phys. 18, 55009 (2016).

16. Nagaosa, N. \& Tokura, Y. Topological properties and dynamics of magnetic skyrmions. Nat. Nanotechnol. 8, 899-911 (2013).

17. Jiang, W. et al. Direct observation of the skyrmion Hall effect. Nat. Phys. 13 162-169 (2017).

18. Litzius, K. et al. Skyrmion Hall effect revealed by direct time-resolved X-ray microscopy. Nat. Phys. 13, 170-175 (2017).

19. Chen, G. Spin-orbitronics: skyrmion Hall effect. Nat. Phys. 13, 112-113 (2017).

20. Barker, J. \& Tretiakov, O. A. Static and dynamical properties of antiferromagnetic skyrmions in the presence of applied current and temperature. Phys. Rev. Lett. 116, 147203 (2016).

21. Zhang, X., Zhou, Y. \& Ezawa, M. Magnetic bilayer-skyrmions without skyrmion Hall effect. Nat. Commun. 7, 10293 (2016).

22. Jin, C., Song, C., Wang, J. \& Liu, Q. Dynamics of antiferromagnetic skyrmion driven by the spin Hall effect. Appl. Phys. Lett. 109, 182404 (2016).

23. Kim, S. K., Lee, K.-J. \& Tserkovnyak, Y. Self-focusing skyrmion racetracks in ferrimagnets. Phys. Rev. B 95, 140404 (2017).

24. Jungwirth, T., Marti, X., Wadley, P. \& Wunderlich, J. Antiferromagnetic spintronics. Nat. Nanotechnol. 11, 231-241 (2016)

25. Montoya, S. A. et al. Tailoring magnetic energies to form dipole skyrmions and skyrmion lattices. Phys. Rev. B 95, 24415 (2017).

26. Nagaosa, N., Yu, X. Z. \& Tokura, Y. Gauge fields in real and momentum spaces in magnets: monopoles and skyrmions. Philos. Trans. R. Soc. A 370, 5806-5819 (2012)

27. Yamane, Y. \& Sinova, J. Skyrmion-number dependence of spin-transfer torque on magnetic bubbles. J. Appl. Phys. 120, 233901 (2016).

28. Radu, I. et al. Transient ferromagnetic-like state mediating ultrafast reversal of antiferromagnetically coupled spins. Nature 472, 205-208 (2011).

29. Meynell, S. A., Wilson, M. N., Fritzsche, H., Bogdanov, A. N. \& Monchesky, T. L. Surface twist instabilities and skyrmion states in chiral ferromagnets. Phys. Rev. B 90, 14406 (2014)

30. Zhang, X., Zhou, Y. \& Ezawa, M. Antiferromagnetic skyrmion: stability, creation and manipulation. Sci. Rep. 6, 24795 (2016).

31. Yang, S.-G., Liu, Y.-H. \& Han, J. H. Formation of a topological monopole lattice and its dynamics in three-dimensional chiral magnets. Phys. Rev. B 94, 54420 (2016).

32. Weindler, T. et al. Magnetic damping: domain wall dynamics versus local ferromagnetic resonance. Phys. Rev. Lett. 113, 237204 (2014).

33. Gerrits, T., Schneider, M. L., Kos, A. B. \& Silva, T. J. Large-angle magnetization dynamics measured by time-resolved ferromagnetic resonance. Phys. Rev. B 73, 94454 (2006)

34. Min, H., McMichael, R. D., Donahue, M. J., Miltat, J. \& Stiles, M. D. Effects of disorder and internal dynamics on vortex wall propagation. Phys. Rev. Lett. 104, 217201 (2010)

35. Lin, S.-Z., Reichhardt, C., Batista, C. D. \& Saxena, A. Dynamics of skyrmions in chiral magnets: dynamic phase transitions and equation of motion. J. Appl. Phys. 115, 17D109 (2014).

36. Rosch, A. Skyrmions: moving with the current. Nat. Nanotechnol. 8, 160-161 (2013).

37. Porter, D. G. \& Donahue, M. J. OOMMF user's guide, version 1.0. NISTIR 6376 (National Institute of Standards and Technology, Gaithersburg, 1999)

38. Liu, L., Lee, O. J., Gudmundsen, T. J., Ralph, D. C. \& Buhrman, R. A. Currentinduced switching of perpendicularly magnetized magnetic layers using spin torque from the spin hall effect. Phys. Rev. Lett. 109, 96602 (2012).

\section{Acknowledgements}

This work was primarily supported by Samsung Research Funding Center of Samsung Electronics under Project Number SRFC-MA1602-01. Part of this work was performed at the PolLux (X07DA) beamline of the Swiss Light Source. S.W. and J.W.C. acknowledge the support from KIST Institutional Program. K.M.S acknowledges the support from the Sookmyung Women's University BK21 Plus Scholarship. X.Z. was supported by JSPS RONPAKU (Dissertation Ph.D.) Program. Y.Z. acknowledges the support by the President's Fund of CUHKSZ, the National Natural Science Foundation of China (Grant No. 11574137), and Shenzhen Fundamental Research Fund (Grant Nos.

JCYJ20160331164412545 and JCYJ20170410171958839). M.E. acknowledges the support by the Grants-in-Aid for Scientific Research from JSPS KAKENHI (Grant Nos. JP17K05490, 25400317, and JP15H05854), and also the support by CREST, JST (Grant No. JPMJCR16F1). S.F acknowledges the support by the EU Horizon 2020 MAGicSky project (Grant No. 665095). K.M.S. and J.W.C. acknowledge the travel fund supported by the National Research Foundation of Korea (NRF) funded by the MSIP (2016K1A3A7A09005418). S.-Y.P. and B.-C.M. acknowledge the support from the National Research Council of Science \& Technology (NST) grant (No. CAP-16-01-KIST) by the Korea government (MSIP). S.-Y.P. also acknowledges the support from KBSI Grant (D37614). S.W. also acknowledges S. Emori for his helpful comments on the manuscript. 


\section{Author contributions}

S.W. designed and initiated the study. K.M.S. optimized structure, fabricated devices, and performed the film characterization. S.W., K.M.S., S.F., and J.R. performed X-ray imaging experiments using STXM at Swiss Light Source in Villigen, Switzerland. X.Z. and Y.Z. performed the numerical simulations. M.E. carried out the theoretical analysis. During the revision of this article, N.J.L., S.-I.K., and S.-Y.P. performed FMR. D.L. and O.L. performed ST-FMR, and S.W., K.M.S., Y.H.K., J.-Y.K., and J.W.C. performed XMCD spectroscopy at $2 \mathrm{~A}$ beamline at Pohang Accelerator Laboratory in Pohang, Korea. S.W., X.Z., and M.E. drafted the manuscript and revised it with assistance from X.L., D.L., O.L., S.-Y.P., J.W.C., B.-C.M., H.C.K., and J.C.. All authors commented on the manuscript.

\section{Additional information}

Supplementary Information accompanies this paper at https://doi.org/10.1038/s41467018-03378-7.

Competing interests: The authors declare no competing interests.

Reprints and permission information is available online at http://npg.nature.com/ reprintsandpermissions/
Publisher's note: Springer Nature remains neutral with regard to jurisdictional claims in published maps and institutional affiliations.

(c) (i) Open Access This article is licensed under a Creative Commons Attribution 4.0 International License, which permits use, sharing, adaptation, distribution and reproduction in any medium or format, as long as you give appropriate credit to the original author(s) and the source, provide a link to the Creative Commons license, and indicate if changes were made. The images or other third party material in this article are included in the article's Creative Commons license, unless indicated otherwise in a credit line to the material. If material is not included in the article's Creative Commons license and your intended use is not permitted by statutory regulation or exceeds the permitted use, you will need to obtain permission directly from the copyright holder. To view a copy of this license, visit http://creativecommons.org/ licenses/by/4.0/.

(C) The Author(s) 2018 